\title{
Discussion sur l'Exposé du Professeur Triffin
}

\section{Monsieur Georges MARTIN *}

L'exposé que nous venons d'entendre, si brillant et parfaitement documenté, dépasse de loin, par les problèmes économiques et financiers qu'il évoque, le cadre purement économique de l'assurance.

Dans le concert économique général où l'industrie et le commerce assument la fonction primordiale, l'assurance a un rôle second à jouer - je n'ai pas dit secondaire - en ce sens qu'elle garantit aux premiers la sécurité financière des personnes, des biens, ainsi que des produits qu'ils livrent, leur permettant ainsi d'exercer leur activité propre avec une tranquillité suffisante. Par sa fonction d'investisseur institutionnel, collecteur d'épargne à long terme, l'assurance procure aux agents économiques, comme à l'Etat d'ailleurs, les moyens financiers appropriés. C'est dans une conjonction dynamique de tous les agents économiques réalisant les adaptations inexorables au sein d'un marché élargi que, sous l'impulsion et le contrôle du Parlement européen, l'Europe fera face aux changements. Les assureurs se doivent de répondre " présents 》.

Le Professeur Triffin vient de nous exposer, avec sa clarté et sa compétence habituelles, les conditions d'une réforme monétaire susceptible d'assurer une meilleure stabilité des monnaies. Il a montré comment l'ECU, se substituant au dollar et au D.T.S., pouvait assumer ce rôle. Il nous a annoncé la constitution d'un Fonds monétaire européen (F.M.E.) de 30 milliards, à la formation duquel ont contribué l'ensemble des pays européens par le versement de $20 \%$ de leurs fonds propres, ceci, dit-il, n'étant qu'un début. Et c'est là que j'émets un certain doute, car pour réaliser une véritable Union monétaire ne faut-il pas une autorité politique et il faut avouer qu'elle est, à l'heure actuelle, précaire ou diffuse.

Certes, dans le passé, notamment au XIXe siècle, il y a eu des unions politiques, mais elles se sont faites sous la contrainte. Aujourd'hui, dans le Marché Commun, on cherche une union par la concertation, ce qui est peut-être un bien, mais qui demande $\mathrm{du}$ temps, beaucoup de temps. C'est pourquoi, personnellement, je ne vois guère l'ensemble des pays du Marché Commun se défaire avant longtemps des $80 \%$ de leurs fonds propres.

J'en viens à l'assurance : c'est évidemment à la stabilité, monétaire tout autant que politique et sociale, que nous aspirons. Internationale par vocation, notamment à travers la technique de la réassurance, l'assurance appelle de tous ses vœux la stabilité des taux de change, seule capable de permettre de voir clair et d'apprécier correctement les équilibres techniques et financiers indispensables.

La variation relative continuelle des monnaies dans le monde, la distorsion dans le temps des engagements des assurés, représentés par les primes qu'ils paient dans une monnaie à un moment donné, et des engagements des assureurs qui sont de payer les sinistres, c'est-à-dire le coût des événements au moment où ils surviennent et dans la monnaie du moment, posent aux assureurs des problèmes financiers et techniques difficiles. C'est pourquoi nous ne pouvons qu'applaudir aux tentatives qui s'élaborent

\footnotetext{
* Vice-Président de 1'« Association de Genève ».
} 
en Europe. Nous ne pouvons que souhaiter que l'ECU remplisse le rôle qui lui est dévolu et que les autorités de chaque pays de la C.E.E. nous autorisent un jour à souscrire des polices en ECU.

Mais, Professeur Triffin, vous l'avez dit vous-même : «Il faut que les Etats-Unis entrent dans le jeu ; s'ils n'entrent pas dans le jeu, nous ne serons nulle part ». Autrement dit, au stade actuel du développement des échanges, le problème monétaire ne peut se régler qu'au niveau mondial.

\section{Monsieur Robert TRIFFIN}

Je suis entièrement d'accord avec l'optique que vient de nous présenter Monsieur Martin et certainement avec sa première remarque : pour réussir dans la voie de l'Union monétaire, il faudra une autorité politique, il faudra effectuer un saut politique. Ça ne fait aucun doute. Mais d'autre part, il est bien certain également que déjà dans le fonctionnement actuel de la Communauté, les décisions ne sont pas simplement économiques. Quand on fixe le prix des denrées agricoles, par exemple, il s'agit d'une décision essentiellement politique. C'est de cela qu'est faite la politique de la plupart de nos pays.

Donc, certainement, il faudra concrétiser cette union politique d'une manière plus solide, plus efficace qu'elle ne l'est aujourd'hui si on veut aller jusqu'au terme de l'opération, c'est-à-dire l'union monétaire. D'autre part, en ce qui concerne le stade actuel, qui n'est pas encore celui de l'union monétaire, mais simplement un système monétaire, la nécessité d'une autorité politique fédérale ou confédérale est moins évidente. Ce serait très souhaitable et peut-être rendu possible, davantage possible, par l'élection du Parlement européen, mais chaque monnaie conservera encore ses fluctuations vis-à-vis de l'écu pendant un certain temps. Il n'y a pas encore de stabilité monétaire irrévocable.

Un second point. Vous avez demandé si les pays sont prêts à se défaire des autres $80 \%$ de leurs réserves monétaires. Vous avez souligné là une erreur sur les mots qui a joué un très grand rôle dans les hésitations, les longues hésitations qu'ont eues certains pays - notamment l'Allemagne - à entrer dans le système monétaire européen. On a présenté ce Fonds monétaire européen comme une sorte de " pool», comme une fusion des réserves de divers pays. Je voudrais répéter ce que j’ai toujours dit : ce n'est pas une fusion des réserves monétaires. Même si tout le monde versait l'intégralité de ses réserves au Fonds monétaire européen, cela ne veut pas dire que la France pourrait tirer sur le compte de l'Allemagne ou inversement. Cela veut simplement dire que tous les pays déposeraient leurs réserves monétaires auprès d'une institution commune gérée par eux au lieu de détenir leurs réserves monétaires, comme c'est le cas aujourd'hui, en obligations du trésor américain ou en dépôts auprès de quelques grandes banques américaines sur lesquelles ils n'ont aucune influence. Il ne s'agit pas d'une fusion des réserves; il s'agit d'une gestion commune. Donc c'est moins inacceptable qu'il ne paraît à première vue.

Alors, est-ce que l'écu est véritablement l'ultime réponse ? $\mathrm{Je}$ ne le crois pas. Je crois que finalement ce sont les monnaies, les diverses monnaies nationales qui se définiront en termes d'écu et qui, à terme, seront remplacées par l'écu. Comment se définira l'écu lui-même? Mais finalement comme disait Gertrude Stein, l'écu sera 
un écu, un écu, comme une rose est une rose, une rose... C'est déjà ce qu'est le dollar, c'est ce que sont toutes les monnaies du monde, et c'est ce qu'était le dollar même sous le prétendu étalon-or qui a survécu officiellement jusqu'en 1971. Le dollar était théoriquement défini en or, et théoriquement, il y avait un certain rapport entre les réserves d'or que devaient conserver les Etats-Unis et le montant total de billets de banques ou de dépôts de la Réserve Fédérale. Il n'y a pas eu de problème aussi longtemps que les réserves américaines furent largement excédentaires. Mais chaque fois que l'on a approché de la limite, on a modifié la loi. Vous savez qu'il existe encore aujourd'hui aux Etats-Unis une loi qui veut que la dette publique des Etats-Unis ne puisse pas excéder tant de centaines de milliards de dollars, et vous voyez qu'en juin de chaque année, il y a une nouvelle proposition au Cóngrès d'augmenter la limite. Et ça passe comme une lettre à la poste, on n'en parle quasi même plus dans les journaux.

Je crois que c'est une illusion de croire que l'or ait jamais été véritablement, en tout cas dans l'après-guerre ou même dans l'après-première guerre, un véritable élément modérateur. Il ne l'était que dans une mesure bien faible. Même lorsque la plupart des pays étaient encore à l'étalon-or, ça n'a pas empêché une inflation, une inflation effrénée. Je voudrais revenir à cet égard sur les longues discussions que j'ai eues avec Monsieur Rueff dont l'amitié m'honorait beaucoup. Nous étions à $100 \%$ d'accord sur les défauts du système d'étalon-or de change, système de dollar-or qui, nous le prédisions, deviendrait inévitablement du dollar papier. Mais nous différions sur le remède. Monsieur Rueff souhaitait revenir à l'étalon-or. Je ne sais pas exactement ce que cela voulait dire, parce que l'étalon-or n'est pas ce que l'on dit dans les manuels. Je croyais que c'était impossible, que l'on devrait aller vers une organisation concertée des réserves monétaires internationales du genre Droits de Tirage Spéciaux, mais avec un nom moins horrible que celui-là. Je voudrais dire pourquoi.

Je suis d'accord avec les économistes pour dire qu'il est absurde de faire dépendre la base des divers systèmes monétaires nationaux du monde de nouvelles découvertes d'or, de leur épuisement ou de la spéculation ou de nouvelles utilisations de l'or qui n'étaient pas prévues hier. C'est idiot, mais à vrai dire lorsque l'homme a prétendu remplacer cette base par une monnaie internationale ou nationale créée par lui, cela a été pire encore. Donc sur ce point-là, si vous voulez, j'étais tenté d'être d'accord avec Monsieur Rueff. Mais malheureusement je crois que sa solution était impossible pour la raison suivante : ce qui faisait la force de l'étalon-or, ou de ce qu'on appelait étalon-or au XIX ${ }^{\mathrm{e}}$ siècle, c'était en fait l'ignorance. C'est que les gouvernements ne s'étaient pas encore rendu compte de leur capacité d'imposer au public des masses énormes de papier-monnaie. Cette ignorance, et là je crois que vous serez d'accord avec moi, n'existe plus, et il n'est pas possible de la recréer. Nous pouvons déplorer le fait que l'on a découvert la bombe atomique, mais elle existe et il n'est pas possible d'effacer de la mémoire de l'homme une chose qu'il a découverte. Si un gouvernement demain acceptait de se lier les mains derrière le dos en disant « moi je reviens à l'étalon-or, on n'émet plus de papier », combien de temps cela durerait-il ? Le même gouvernement, ou le suivant, saurait qu'il peut revenir au papier pour résoudre ses problèmes économiques ou politiques. L'ignorance ne peut pas être recréée et c'est pour ça que la solution de Monsieur Rueff - aussi désirable qu'elle puisse sembler n'est pas à mon avis praticable. 
Finalement, vous avez dit, et c'est très juste, que si les Etats-Unis n'entrent pas dans le jeu, les problèmes du système monétaire européen seront énormes. C'est vrai, mais d'autre part, le système monétaire européen a été créé précisément à cause du fait que les Etats-Unis n'étaient pas entrés dans le jeu des réformes monétaires mondiales. On a toujours créé, on a toujours avancé sous l'influence d'une menace externe. Je me souviens au temps où j'ai aidé à créer l'Union Européenne des Paiements, c'était la menace russe. Aujourd'hui c'est la menace de l'inflation du dollar qui a finalement forcé les Européens à agir. J'espère et je crois fondamentalement - ce n'est pas seulement une espérance, mais une conviction - que le système monétaire européen devrait aider l'Europe à coopérer avec les Etats-Unis afin de rendre la santé indispensable au dollar et je crois que c'est dans cette voie-là que nous marcherons à l'avenir.

Mais d'autre part, si on ne faisait pas cela, si l'Europe ne coopérait pas, ou si les Etats-Unis ne coopéraient pas, cela ne voudrait pas dire que le système passé serait préférable au système monétaire européen. Ce dernier apparaîtrait alors comme un pis aller, une sorte de palliatif à l'instabilité mondiale déclenchée par le refus des Etats-Unis. Ça ne voudrait pas dire que le système monétaire européen ne pourrait pas fonctionner. Il serait encore plus nécessaire, si vous voulez, bien que moins efficace.

\section{Monsieur le Commissaire Etienne DAVIGNON *}

Je vais faire une remarque très brève à propos d'un problème évoqué par Messieurs Triffin et Martin, problème qui me paraît particulièrement important. C'est vrai que nous avons fait au niveau européen un pari extraordinaire qui consiste à anticiper une réalité. C'est vrai que l'on peut faire un discours extraordinaire pour expliquer que l'Europe n'existe pas, mais on peut arriver à ébranler un certain nombre de lacunes évidentes. Si l'on analyse la période de progrès de l'Europe par rapport à ses périodes de stagnation, on trouve toujours un fil conducteur. Pourquoi est-on en train de parler de l'Union monétaire ou du système monétaire aujourd'hui ? C'est parce qu'on n'a pas réussi à le faire dans des circonstances qui étaient plutôt plus favorables. La pression des besoins n'était pas la même.

Si chacun d'entre nous aujourd'hui se dit qu'il ne va accomplir une action que dans la mesure où il a la certitude que, quelque soient les aléas, c'est une bonne décision, rien n'est accompli. Or je suis persuadé que les progrès du système monétaire européen sont directement liés aux usages que les opérateurs économiques, pour prendre le terme le plus général que je puisse trouver, vont en faire. C'est comme ça qu'on casse le scepticisme. A partir du moment où il y a quelqu'un qui a pris une décision, un grand nombre d'autres personnes se disent, tiens pourquoi a-t-il pris cette décision ? Si lui, qui est plutôt raisonnable, s'engage dans cette voie-là, c'est qu'il ne la considère pas comme excessivement risquée.

Pour prendre un exemple très simple, quand la sidérurgie était sur le point de disparaître ou de devenir un service public au même titre que les tramways, on est parti de l'idée qu'il fallait lutter contre cette situation. Si à ce moment les opérateurs avaient estimé que la difficulté de l'entreprise était telle que ça ne valait pas la peine, une autorité dictatoriale européenne aurait échoué.

Nous sommes dans une période de sociétés économiques dans laquelle la réalité

* Membre de la Commission des Communautés Européennes, Bruxelles. 
repose sur le consentement du plus grand nombre, comme dans le cas du système bancaire. Or, je crois que dans la situation qui est la nôtre, l'acceptation du changement et son utilisation dans un sens positif constituent un moins grand risque qu'une attitude d'attentisme. Car le changement finira par s'imposer, mais dans de mauvaises conditions.

\section{Monsieur Orio GIARINI}

Il n'est pas rare aujourd'hui d'entendre affirmer que l'assurance ne joue dans l'économie qu'un rôle accessoire, voire secondaire. Une opinion aussi surprenante s'explique peut-être par le fait que les assureurs sont généralement des gens discrets qui pratiquent volontiers l'understatement. Pourtant il suffit de regarder les chiffres pour se rendre compte que, de toute évidence, l'assurance joue en fait un rôle essentiel dans l'économie. Pour nous en convaincre, il suffit de nous rappeler que, dans les pays industrialisés, le total des primes d'assurance équivaut à un pourcentage du revenu national avoisinant 6 à $7 \%$ ou encore qu'en Grande-Bretagne, par exemple, l'assurance est essentielle pour l'équilibre de la balance des paiements. Dans d'autres domaines également, nous voyons apparaître des signes qui augurent du rôle toujours plus important de l'assurance dans le monde. Ainsi, on constate actuellement dans la plupart des pays une crise de plus en plus profonde des systèmes de sécurité sociale et ce phénomène oblige les individus et l'économie dans son ensemble à redécouvrir les avantages de la protection contre les risques par le biais des institutions de type pluraliste. Devant cette évolution, il me paraît tout à fait légitime de prévoir que, d'ici dix ou quinze ans, l'assurance organisée sur une base pluraliste pourrait représenter jusqu'à $10 \%$ du revenu national en termes de primes encaissées.

J'aimerais poser à présent deux questions. Tout d'abord, j'aimerais demander au vicomte Davignon quel poids politique il attribue à l'élection du Parlement européen en tant qu'élément susceptible de contribuer au développement économique.

Pour ce qui est du problème monétaire, j'aimerais que soient précisées les possibilités qu'a l'ECU de devenir une monnaie de réserve sur le plan mondial.

En effet, on constate actuellement une demande importante en monnaies fortes (marks allemands et francs suisses). Cette tendance se manifeste même au niveau des banques centrales de certains pays d'Asie. Or, la surface économique d'un pays comme l'Allemagne et, à plus forte raison, comme la Suisse est tout de même relativement restreinte par rapport à l'échelle mondiale. On peut donc très bien imaginer que pendant un certain temps le marché mondial absorbera toute "production » nouvelle de monnaies fortes avec pour seul résultat un accroissement de la demande et non un affaiblissement des monnaies trop fortes. Nous en arrivons donc à penser que plus une monnaie ou une économie forte se renforce, plus elle devient vulnérable. Il faut ajouter à ce tableau le poids du système mondial qui restreint les marges de manœuvre.

C'est pourquoi, je crois qu'il serait intéressant de nous interroger sur les possibilités de l'ECU et d'examiner en particulier dans quelle mesure il pourrait contribuer à améliorer la situation mondiale, en devenant une monnaie de réserve pour toutes les banques centrales.

\section{Monsieur le Commissaire Etienne DAVIGNON}

Je vais être très bref sur la question qui a été posée. Je crois que dans le mouvement de dynamisme indispensable que doit connaître l'action concertée des Etats dans 
le cadre de la communauté, et je choisis à dessein mes mots, le rôle du Parlement est à la fois important et secondaire. Il est important parce qu'il permet d'apporter la preuve que l'évolution de l'Europe n'est pas indifférente par rapport au rôle du citoyen de chacun de nos pays. Dans la mesure où l'Europe apparaît comme étant une réalité distincte, je dirais une réalité touchant plus à la politique extérieure qu'à la réalité vive de ce qu'est l'avenir de chacun d'entre nous, il est clair qu'il est difficile à la fois d'expliquer ce que l'on essaie de faire et de justifier l'ambition que l'action doit avoir. Le rôle du Parlement est important. Mais si l'on pense que c'est le Parlement qui va devenir le moteur de l'opération, c'est aussi faux que de croire que la Commission européenne va devenir le moteur de l'opération ou de croire que l'un ou l'autre grand Etat va devenir le moteur de l'opération. Parce que ce que nous avons constaté, c'est que personne n'a tout seul le pouvoir de concevoir une politique, de l'exécuter, et de la faire réussir. La politique est quelque chose qui requiert une notion de succès minimal. Vous savez bien que dans chacune de vos opérations, si vous ne pouviez pas faire apparaître à la fin de l'année, au moment de votre assemblée générale, que la voie que vous avez choisie n'est peut-être pas aussi pleine de succès que vous l'aviez espéré mais que, dans le bilan général que vous apportez, il y a des réalisations positives, vous vous trouveriez même avec les meilleures des idées devant une situation difficile. Et je crois que c'est très important de savoir cela. Nous ne pourrons faire les choses que de la manière la plus difficile, c'est-à-dire avec un consensus suffisant de ceux qui ont une influence sur les événements ; nous ne pourrons pas les faire sans les opérateurs économiques, nous ne pourrons pas les faire sans les forces du travail, nous ne pourrons pas les faire sans les forces du capital et nous ne pourrons pas les faire sans les Etats. Mais nous ne pourrons pas les faire avec l'une de celles-là toute seule et le Parlement ne peut pas plus vrendre le relais que qui que ce soit d'autre. C'est donc un élément important pour faire passer le message, un élément important pour démontrer que l'exigence n'est pas une exigence abstraite, une exigence de visionnaire ou de personne illuminée, mais qu'elle correspond véritablement à la seule alternative concrète et visible de réduire les insécurité et les incertitudes auxquelles on est confronté.

En ce sens-là, le Parlement est important, mais il n'est pas décisif comme je le crains - aucun élément n'est décisif - c'est ce qui fait de la tâche qui est la nôtre une tâche tellement importante parce que personne ne peut s'en désintéresser, parce que toute pe:sonne qui s'en désintéresse prend par le fait même une part de responsabilité dans l'inadéquation de la réponse donnée à la question que personne ne peut contester, se poser dans la réalité des choses. Je crois que c'est la difficulté de la situation.

\section{Monsieur Robert TRIFFIN}

Je plaide depuis 1959 pour la création d'une monnaie de réserve autre qu'une monnaie nationale, que le dollar à cette époque. Mais ceci dit, évidemment vous avez raison : pour avoir une véritable monnaie de réserve mondiale, il faut quelque chose de plus que l'écu. Il faudrait un DTS, mais totalement transformé évidemment, et c'est là où la coopération des Etats-Unis est indispensable pour la réussite de cette réforme mondiale. Au stade où nous en sommes, et faute d'un tel accord jusqu'ici, le mieux que nous puissions faire c'est d'avoir au moins une monnaie de change européenne, pas encore une véritable monnaie, une monnaie qui circule dans les transactions internes. Ce qu'il faut c'est que l'écu puisse servir aux transactions extérieures, que l'on puisse s'en servir aussi bien, ou peut-être même mieux, que du dollar. Je crois que c'est préci- 
sément là ce qui a conduit les Allemands, notamment, à entrer dans cette voie. En effet, ce qui a absolument effaré les Allemands, c'est que toutes les difficultés du dollar se traduisaient par des mouvements massifs d'argent vers le mark ou le franc suisse. Or ni la Suisse ni l'Allemagne n'ont envie de faire suivre à leur monnaie nationale le sort qui a été celui de la livre, dont le rôle de monnaie de réserve a détruit la convertibilité en septembre 1931, quarante ans presque jour pour jour avant que le dollar suive le même sort. Et je souhaiterais d'ailleurs que le jour où l'on pourra avoir une véritable réforme monétaire mondiale, l'écu ne suive pas non plus cette voie. L'écu ne doit pas devenir une monnaie de réserve mondiale, mais dans la période intermédiaire que nous vivons maintenant, l'écu serait une meilleure monnaie de réserve, qui créerait moins de difficultés que des monnaies de réserve nationales.

Comme vous le savez, les banques centrales des pays de la Communauté ne conservent pas de réserves dans la monnaie d'un autre pays de la Communauté. L'écu leur permettra de détenir des réserves autres que le dollar sans pour cela devoir détenir des monnaies nationales de pays de la Communauté. C'est dans cette voie-là que nous sommes engagés.

Enfin, je voudrais dire encore un mot, bien qu'on ne m'ait pas posé cette question. Il est bien certain que tout cela ne pourra réussir que si les secteurs privés y mettent du leur, s'ils se mettent dans le bain comme les autres, comme le disait très bien également le Vicomte Davignon. Je suis très encouragé par le fait que certaines grandes banques, en Belgique et en France aussi d'ailleurs, entrent dans cette voie, commencent à utiliser l'écu comme dénominateur commun pour certaines de leurs opérations ; les sociétés d'assurance évidemment ont tout intérêt à faire exactement la même chose et si nous pouvons avoir la coopération des banques privées, des sociétés d'assurance, je crois que nous sommes dans la bonne voie.

\section{Monsieur Claude BEBEAR}

Je voudrais faire remarquer qu'un certain nombre de compagnies d'assurances souscrivent déjà des emprunts exprimés en euro devises.

Je crois que si on leur proposait des emprunts souscrits en écu, elles y souscriraient à condition bien entendu qu'on prévoit ce qu'il adviendrait en cas de disparition de l'écu.

Vous revenez, nous avez-vous dit, des pays arabes. Est-ce que les pays arabes seraient disposés à ce qu'on leur paie leur pétrole en écu?

\section{Monsieur Robert TRIFFIN}

Je crois que c'est une hypothèse qui vient seulement de commencer à être envisagée. Dans l'immédiat, ils songent surtout à commercialiser le pétrole en Droits de Tirages Spéciaux plutôt qu'en dollars. Le DTS pour eux a encore un sens parce que les pays arabes ont évidemment des relations extrêmement importantes avec les Etats-Unis. Le fait que le dollar joue un rôle peut-être excessif dans la définition du DTS ne les inquiète pas autant que cela inquiéterait les pays européens. Mais d'autre part à choisir entre le DTS et l'écu, n'est-ce pas, il y a toute une série de considérations que je n'aurais pas le temps de développer en ce moment, mais j'en indiquerai au moins deux si vous voulez. 
L'une c'est que pour tous les banquiers privés, l'utilisation de l'écu est beaucoup plus facile que l'utilisation du DTS parce que le DTS c'est 16 monnaies et pour certaines de ces monnaies, il n'y a pas de marché monétaire national dans lequel les banques peuvent investir. L'écu c'est un nombre beaucoup moindre de monnaies avec partout des marchés monétaires parfaitement disponibles. Ils les utilisent largement et facilement.

Un autre point, qui est extrêmement important, c'est que lorsque l'on a adopté le DTS initialement, on a dit dans le premier amendement aux Statuts du Fonds Monétaire International que le DTS était un certain poids d'or. Mais le jour où le dollar est devenu inconvertible, en août 1971, soudainement cette définition a disparu. Le DTS est devenu, pendant plusieurs mois, simplement du dollar papier. Il a été identifié au dollar fluctuant. Après cela, on a changé la définition; on a fait du DTS un panier de monnaies, mais tout cela était parfaitement illégal.

Pour prendre ces décisions, il aurait fallu des majorités considérables. Au Fonds monétaire on a pris ces décisions à 10 ou à 15 , dans l'illégalité la plus totale. Le seul mérite du second amendement des statuts du FMI, à mon sens, a été de légaliser, après des années, l'illégalité qui s'était généralisée dès 1971 et 1973 . On a légalisé ce qui existait ; et ce qui existait, c'est que tous les pays avaient répudié illégalement leurs obligations, les engagements qu'ils avaient pris à Bretton Woods. C'est là un autre argument qui incite à aller vers l'écu. On craint que le DTS ne soit de nouveau sujet à de telles modifications, légales ou illégales, alors que c'est beaucoup moins probable en ce qui concerne l'écu. Notamment, les pays européens qui ont la charge de l'écu préfèrent avoir leur mot à dire plutôt que d'être totalement subordonnés à des décisions qui pourraient être prises à la manière dont les décisions ont été prises en ce qui concerne le DTS. Mais à part ça, je crois qu'à long terme, il faudra revenir à une véritable réforme monétaire internationale et une nouvelle forme de DTS, comme instrument international mondial de réserves, qui prenne la place du dollar et des autres monnaies nationales. 